\title{
THE
}

1995

\section{Incipience of quantum chaos in the spin-boson model}

Michel Cibils

Yvan Cuche

Gerhard Müller

University of Rhode Island, gmuller@uri.edu

Follow this and additional works at: https://digitalcommons.uri.edu/phys_facpubs

Terms of Use

All rights reserved under copyright.

\section{Citation/Publisher Attribution}

Michel Cibils, Yvan Cuche and Gerhard Müller. Incipience of quantum chaos in the spin-boson model. Z. Phys. B 97 (1995), 565-572.

Available at: http://dx.doi.org/10.1007/BF01322441

This Article is brought to you for free and open access by the Physics at DigitalCommons@URI. It has been accepted for inclusion in Physics Faculty Publications by an authorized administrator of DigitalCommons@URI. For more information, please contact digitalcommons-group@uri.edu. 


\title{
Incipience of quantum chaos in the spin-boson model
}

\author{
Michel Cibils, ${ }^{1}$ Yvan Cuche, ${ }^{2}$ and Gerhard Müller ${ }^{3}$ \\ 1 Institut de Physique Théorique Ecole Polytechnique Fédérale de Lausanne \\ PHB-Ecublens, CH-1015 Lausanne, Switzerland \\ 2 Istituto Nazionale di Ottica Largo E. Fermi 6, 50125 Firenze, Italy \\ 3 Department of Physics, University of Rhode Island, Kingston RI 02881, USA
}

\begin{abstract}
The peculiar spectral properties of the spin-boson model make it suitable for an investigation of quantum nonintegrability effects and level statistics from a new perspective. For fixed spin quantum number $s$, its energy spectrum consists of $2 s+1$ sequences of levels with no upper bound. These sequences are identified and labelled consecutively by means of a quantum invariant calculated from the time average of a non-stationary operator. For integrable cases, level repulsion (on the energy axis) is limited to states within each sequence. From the observed spectral properties, we infer a series of $s$-dependent level-spacing distributions. They converge towards a Poisson distribution for $s \rightarrow \infty$. For nonintegrable cases, level repulsion becomes a universal phenomenon, but the amount of repulsion between two states decreases with increasing separation (in label) of the two sequences to which they belong. For small $s$, the quantum nonintegrability effects are compelling but not at all chaotic. Nevertheless, they contain all the ingredients necessary to produce the symptoms commonly described as indicators of quantum chaos. In this model, we can observe quantum chaos in the making under very controllable conditions.
\end{abstract}

\section{INTRODUCTION}

The spin-boson model has been investigated in a variety of different contexts (atomic physics, solid state physics, quantum optics) and, until recently, mostly for purposes unrelated to quantum chaos (see references in $[1,2,3,4])$. Existing quantum chaos studies of the spinboson model have focused primarily on properties of the flow in the classical phase space and on quantum mechanical representations thereof (coherent-state representation, Husimi distributions $[3,4,5]$, dynamics of level occupation probabilities [1], and energy-level statistics $[6,7,8]$. The purpose of this study, which is based on the methods of analysis introduced in $[9,10,11]$ for a two-spin system, is the investigation of nonintegrability effects in quantum invariants of the spin-boson model and their implications for the level statistics. The spin-boson Hamiltonian considered in this work reads :

$$
\begin{aligned}
H & =\hbar \omega_{B} a^{\dagger} a+\hbar \omega_{S} S_{z} \\
& +\sqrt{\frac{\hbar}{2}} \hbar \frac{\Lambda}{2}\left\{S_{+} a+S_{-} a^{\dagger}+\epsilon\left(S_{+} a^{\dagger}+S_{-} a\right)\right\},
\end{aligned}
$$

where $a^{\dagger}, a$ are boson creation and annihilation operators with $\left[a, a^{\dagger}\right]=1$ of a quantum harmonic oscillator, and $S_{ \pm}=S_{x} \pm i S_{y}, S_{z}$ are dimensionless operators with $\left[S_{x}, S_{y}\right]=i S_{z}$ etc. of a spin with quantum number $s$.

In the absence of any interaction, both subsystems have equidistant and non-degenerate levels, with spacings $\hbar \omega_{B}$ for the boson levels and $\hbar \omega_{S}$ for the $2 s+1$ spin levels. $\Lambda$ is the spin-boson coupling constant, and $\epsilon$ interpolates between the Jaynes-Cummings model $(\epsilon=0)$ and the standard spin-boson model $(\epsilon=1)$. The Hamiltonian (1) plays an important role in the nonrelativistic theory of interaction between atoms and a radiation field. In that context, the boson represents one mode of the electromagnetic field and the coupling term in (1) its interaction with a $(2 s+1)$-level atom.

In the limit $\hbar \rightarrow 0, s \rightarrow \infty, \hbar s=S=$ const, $H$ turns into the energy function $\mathcal{H}$ of an autonomous classical Hamiltonian system of two degrees of freedom $[2,3$, $12]$ - a classical harmonic oscillator coupled to a threecomponent vector of fixed length $S$. For $\epsilon=0$ only, a second integral of the motion (in addition to $\mathcal{H}$ ) exists, and guarantees integrability. For $\epsilon>0$, the phase flow is chaotic. Chaos is widespread for $\epsilon=1$ and $\Lambda$ sufficiently large $[12,13]$, and becomes increasingly constrained by a growing measure of intact tori as $\epsilon$ approaches zero. This justifies the name integrability parameter for $\epsilon$.

The (formal) quantum counterpart of the second classical integral of the motion is the operator

$$
I=\hbar\left(a^{\dagger} a+S_{z}\right)
$$

which commutes with $H$ only for $\epsilon=0$. However, it is not a priori clear, what the impact of $[H, I] \neq 0$, i.e. $\epsilon \neq 0$, might be on the spectral properties of (1) for small values of the spin, $s=1 / 2$ and $s=1$, in particular. These systems have little in common with two classical degrees of freedom, for which an integrability criterion with clearcut consequences exists. The phenomena of quantum nonintegrability indeed turn out to be dramatic even for small spin quantum numbers. Although distinct from the commonly used indicators of quantum chaos, the observed effects contain the building blocks that produce them. Hence the name incipient quantum chaos. Its manifestations in the spin-boson model with $s=1 / 2, s=$ 1 , and $s>1$ will be discussed in Secs. III, IV and V, respectively, following a brief introduction to the method of analysis in Sec. II. 


\section{QUANTUM INVARIANTS}

For all integrable cases $(\epsilon=0)$, the energy spectrum $E_{\lambda}$ of the spin-boson Hamiltonian (1) consists of $2 s+1$ infinite sequences of levels. The eigenstates $|\lambda\rangle$ are naturally labelled by a pair of quantum numbers $\lambda=(\mu, \nu)$, where $\mu=-s,-s+1, \ldots,+s$ specifies the sequence, and $\nu=0,1,2, \ldots$ the position in that sequence. For the noninteracting case $\Lambda=0$, these two natural quantum numbers are the spin $z$-component $\sigma=\left\langle\lambda\left|S_{z}\right| \lambda\right\rangle \equiv\left\langle S_{z}\right\rangle_{\lambda}$ and the boson occupancy $n=\left\langle a^{\dagger} a\right\rangle_{\lambda}$, respectively.

In the presence of a spin-boson interaction $(\Lambda \neq 0)$, the two operators $S_{z}$ and $a^{\dagger} a$ are no longer invariant under time evolution, but their time averages as represented by the diagonal matrices $\left\{\left\langle S_{z}\right\rangle_{\lambda}\right\},\left\{\left\langle a^{\dagger} a\right\rangle_{\lambda}\right\}$ are new invariant operators. Their matrix elements are then no longer integers or half-integers, but the points with coordinates $\left(\left\langle S_{z}\right\rangle_{\lambda},\left\langle a^{\dagger} a\right\rangle_{\lambda}\right)$ still form $2 s+1$ infinite strands. That is also the case if we plot the matrix elements of an arbitrary operator, $\langle A\rangle_{\lambda}$, versus those of the Hamiltonian, $E_{\lambda}=\langle H\rangle_{\lambda}[9]$. The perfectly regular arrangement of the points $\left(\langle A\rangle_{\lambda}, E_{\lambda}\right)$ reflects a "smooth" dependence of these quantum invariants on two quantized actions.

For nonintegrable cases $(\epsilon \neq 0)$ indeed some dramatic changes in pattern take place in the $\left(\langle A\rangle_{\lambda}, E_{\lambda}\right)$-plane as we shall see. These changes depend strongly on the spin quantum number, at least for small $s$, but they are all connected with the loss of one of the two natural quantum numbers identified above.

This type of quantum chaos study based on invariants determined via time averages of non-stationary operators was introduced in the context of the study of a twospin model with a nontrivial integrability condition for the purpose of establishing a direct link between the destruction of invariant tori in the classical phase flow and the appearance of defects in the configuration of points $\left(\langle A\rangle_{\lambda}, E_{\lambda}\right)[9]$.

Here we use the quantum invariants $\langle A\rangle_{\lambda}, E_{\lambda}$ in order to understand the peculiar level-spacing distributions of the spin-boson model (1). Throughout this study we shall use the operator $A=a^{\dagger} S_{-}$for that purpose, but this choice is by no means essential for the conclusions that will be reached. Even though $a^{\dagger} S_{-}$is not hermitian, the matrix elements $\left\langle a^{\dagger} S_{-}\right\rangle_{\lambda}$ happen to be real for all eigenstates of (1). In the basis built from the eigenstates $|n, \sigma\rangle$ of the noninteracting system (see Sec. II), both $H$ and $a^{\dagger} S_{-}$are real matrices, and the eigenvectors of $H$ have real coefficients. In all our numerical calculations we have set $\hbar=1 / s$, which yields a classical spin length $S=1$ in the limit $s \rightarrow \infty$.

\section{III. $\quad$ SPIN $s=1 / 2$}

The extreme quantum case $(s=1 / 2)$ of the spin-boson model (1) is only very remotely related to a classical system with two degrees of freedom, for which the integrability condition is directly linked to drastic consequences.
One might think that the quantum integrability condition, which is basically a classical notion formally translated into quantum mechanics in the form of the commutativity requirement $[H, I]=0$ of operators (1) and (2), has little if any impact on the spectral properties in the spin-boson model for small $s$, and that nonintegrability effects begin to be noticeable for large $s$ only, where the correspondence principle comes into play. Contrary to this expectation, we demonstrate that integrability and nonintegrability have clear-cut consequences even for $s=1 / 2$, leading, for example, to well-defined and distinct level-spacing distributions. For that demonstration, we investigate the eigenvalues of two quantum invariants as described in Sec. II.

\section{A. $[H, I]=0$}

Figure 1 shows a plot of the invariant $\left\langle a^{\dagger} S_{-}\right\rangle_{\lambda}$ versus the invariant $E_{\lambda}$ (energy) for an integrable case $(\epsilon=0)$ of the $s=1 / 2$ spin-boson model. We observe two strands of states that live in different worlds. The levels within each strand have nearly uniform spacings, but the spacings on one strand are completely unaffected by the levels on the other strand. For a close view, we show in the inset part of the same data on an expanded energy scale and with successive levels connected by dashed lines. The arrow marks one location on the energy axis, where levels from different strands are nearly degenerate. Strong intrastrand level repulsion combined with zero inter-strand level repulsion is the hallmark of integrability in the spinboson model - also for $s>1 / 2$ as we shall see.

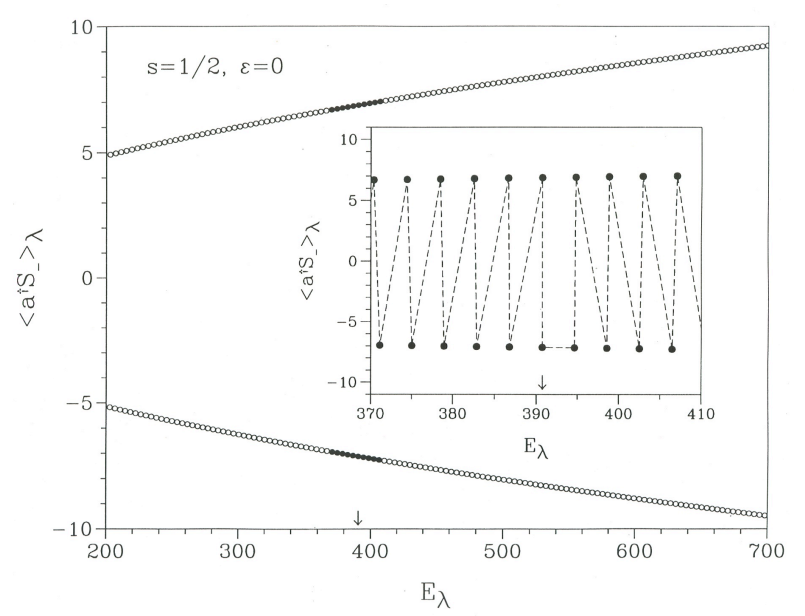

FIG. 1: Invariant $\left\langle a^{\dagger} S_{-}\right\rangle_{\lambda}$ versus energy eigenvalue $E_{\lambda}$ for the eigenstates of parity +1 of the (integrable) $s=1 / 2$ spin-boson model (1) with $\hbar=1 / s=2, \omega_{B}=\omega_{S}=1, \Lambda=1$, and $\epsilon=0$, over an energy range of given size. A subset of these states (full circles) is shown again in the inset on an expanded energy scale and with consecutive levels connected by dashed lines. The arrow indicates a point of inter-strand near-degeneracy. 
What kind of level-spacing distribution does this twostrands configuration of eigenstates produce? Toward a quantitative analysis of that question, we have plotted in Fig. 2 the sequence of successive energy-level spacings, $\Delta E_{\lambda}=E_{\lambda}-E_{\lambda-1}$ versus $E_{\lambda}$ over the same interval as the levels themselves were shown in Fig. 1. Almost all spacings are of the inter-strand type and fall alternatingly onto one of two piecewise smooth curves. They are well approximated by triangular functions with the same amplitude and period, but with opposite phases. The location of one near-degeneracy (the same as in Fig. 1) is again marked by an arrow.

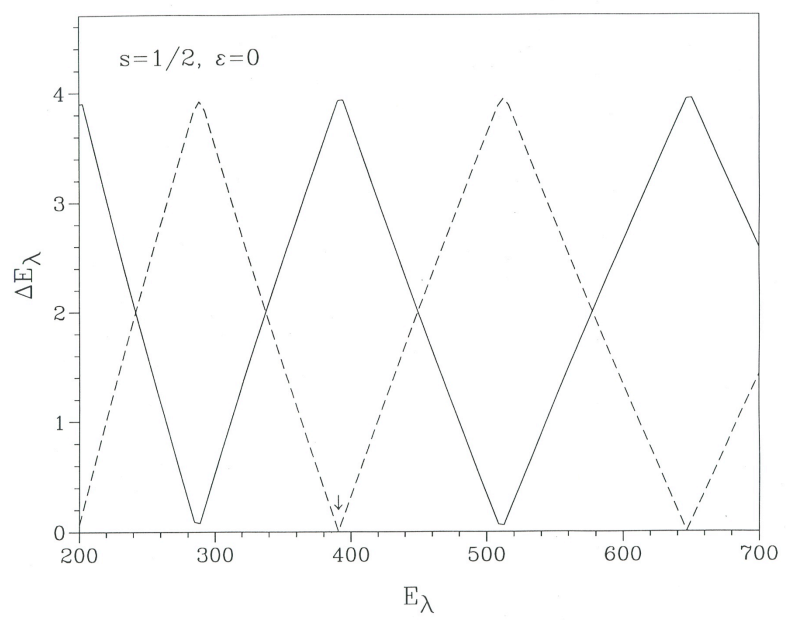

FIG. 2: Sequence of successive spacings $\Delta E_{\lambda}=E_{\lambda}-E_{\lambda-1}$ between the energy levels shown in Fig. 1 for the (integrable) $s=1 / 2$ spin-boson model $(1)$, with $\hbar=1 / s=2, \omega_{B}=\omega_{S}=$ $1, \Lambda=1$, and $\epsilon=0$. The spacings fall alternatingly onto one or the other of two piecewise smooth curves (shown as dashed and solid lines) The arrow indicates the location of the same inter-strand near-degeneracy as in Fig. 1.

If we ignore the slowly varying period and amplitude of the two curves in Fig. 2, the resulting level-spacing distribution is rectangular,

$$
P_{2}(\Delta E)=\frac{1}{\Delta E_{S}} \Theta(\Delta E) \Theta\left(\Delta E_{S}-\Delta E\right) .
$$

The maximum spacing $\Delta E_{S}$ is equal to the intra-strand spacing, here assumed to be nearly the same in both strands. The rectangular function (3) provides a fairly accurate description of the actual spacing distribution of the energy levels for this system as shown in Fig. 3. In order to avoid the strong nonuniformities at the lower end of the spectrum and the inevitable truncation effects that impact upon the highest levels obtained by numerical diagonalization, we use only the levels from a certain intermediate energy interval for the level statistics. Over that interval the density of levels is nearly constant. Therefore no unfolding is necessary in the statistical analysis. The rectangular distribution is characteristic for the integrable $s=1 / 2$ spin-boson model, but only for non-zero interaction $(\Lambda \neq 0)$. In the absence of spin-boson coupling $(\Lambda=0)$, the level spacings become all equal (see $[6])$.

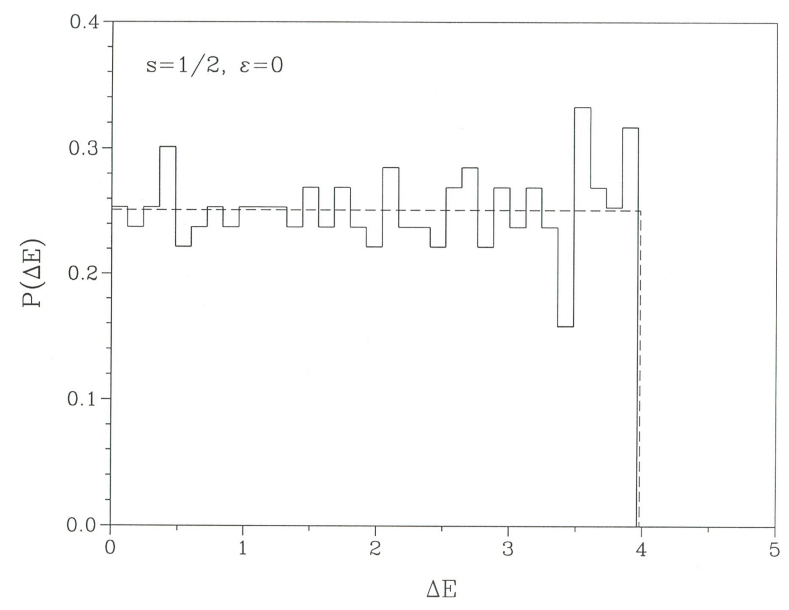

FIG. 3: Level-spacing distribution of the states shown in Fig. 1 , but over the extended energy range $99 \leq E_{\lambda} \leq 1130$ (515 levels), of the (integrable) $s=1 / 2$ spin-boson model (1) with $\hbar=1 / s=2, \omega_{B}=\omega_{S}=1, \Lambda=1$, and $\epsilon=0$ (histogram). The dashed line represents the rectangular distribution (3), which is predicted by a simple phenomenological model as described in the text.

For a simple model of the two-strands level-sequence that characterizes the integrable $s=1 / 2$ spin-boson model, consider two 1D lattices with incommensurable spacings $b_{1}=b<b_{2}$. The superposition of the two lattices yields an inhomogeneous array of sites, and the spacings $x$ between successive sites satisfy the distribution

$$
p_{2}(x)=\left(1-u_{2}\right) \tilde{p}_{2}(x) \Theta(x) \Theta(b-x)+u_{2} \delta(x-b),
$$

where $\tilde{p}_{2}(x)=$ const $=1 / b$, and where the second term represents the fraction of intra-lattice spacings that survive the superposition. For small differences $b_{2}-b_{1}=\eta$ in lattice spacings, we have $u_{2} \propto \eta$. In the spin-boson application of Fig. 3, the peak predicted at the band edge is insignificant relative to the statistical fluctuations in the histogram. The lattice model will be generalized later to describe the level spacings of the integrable $s>1 / 2$ spin-boson model.

\section{B. $[H, I] \neq 0$}

What is the impact of nonintegrability $(\epsilon \neq 0)$ on the configuration of states depicted in Fig. 1 for $\epsilon=0$ ? The answer is shown in Fig. 4. The two strands are still there, but now they "interact" in the vicinity of each interstrand near-degeneracy. This happens at fairly regular intervals and gives rise to a standing-wave like pattern 


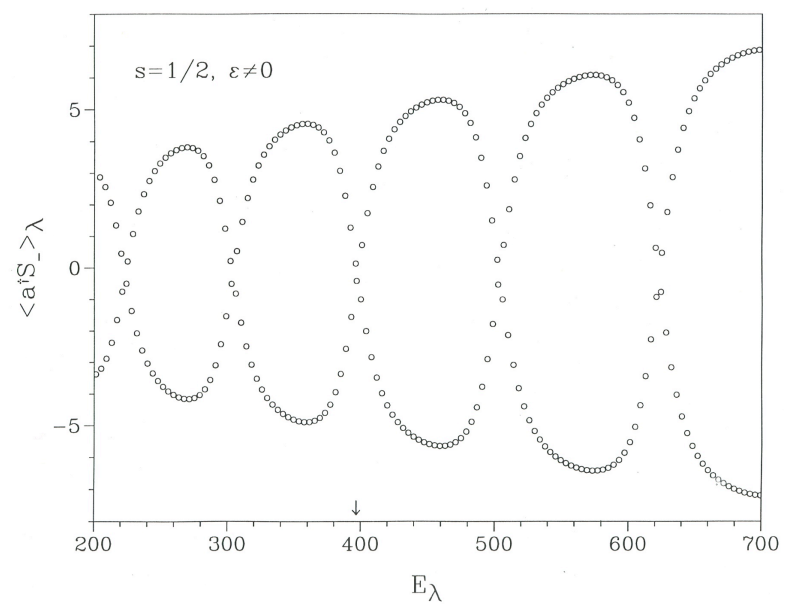

FIG. 4: Invariant $\left\langle a^{\dagger} S_{-}\right\rangle_{\lambda}$ versus energy eigenvalue $E_{\lambda}$ over a certain energy range for the eigenstates of parity +1 of the (nonintegrable) $s=1 / 2$ spin-boson model (1), with $\hbar=1 / s=$ 2, $\omega_{B}=\omega_{S}=1, \Lambda=1$, and $\epsilon=0.7$. The arrow indicates the location of one near-degeneracy between energy levels.

in the configuration of points $\left(\left\langle a^{\dagger} S_{-}\right\rangle_{\lambda}, E_{\lambda}\right)$. One such occurrence in Fig. 4 is marked by an arrow.

This "interaction" between nearly degenerate strand states will be called a resonance. It is a form of hybridization and involves two major effects that are relevant in the context of this study:

(i) The resonating states are physically much less distinct than the original strand states. They produce expectation values $\left\langle a^{\dagger} S_{-}\right\rangle_{\lambda}$ that are more alike than those of pure strand states. In the absence of integrability, there is no longer that second quantum number which keeps states from different strands in different worlds.

(ii) In a resonance, one of the two states involved is pushed to a slightly lower energy and the other one to a slightly higher energy. This level repulsion, although undetectably small on the energy scale of Fig. 4, produces a major change in the level-spacing distribution.

The level repulsion near resonances is best visible in the sequence $\Delta E_{\lambda}$ of successive spacings as shown in Fig. 5 . The spacings fall again, as in Fig. 2, alternatingly onto one of two nearly periodic curves, but now these curves have round minima and maxima, and the minima are nonzero by a significant amount. Very small spacings are eliminated completely by the resonances.

In an idealized model for the observed level-spacing sequences, we may approximate the two curves in Fig. 5 by two simple harmonic functions with equal amplitudes and periods, but opposite phases. Under the assumption that the density of levels is constant on each strand, this

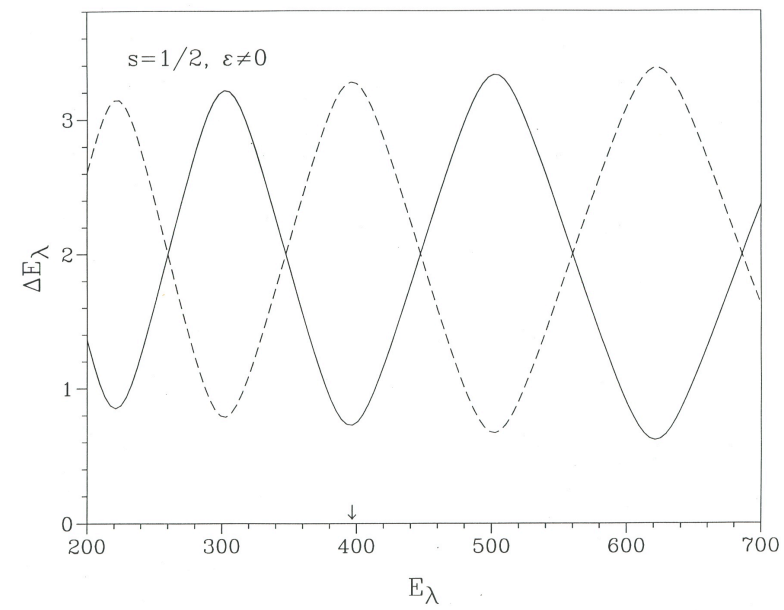

FIG. 5: Sequence of successive spacings $\Delta E_{\lambda}=E_{\lambda}-E_{\lambda-1}$ between the energy levels shown in Fig. 4 for the (nonintegrable) $s=1 / 2$ spin-boson model (1), with $\hbar=1 / s=2, \omega_{B}=\omega_{S}=$ $1, \Lambda=1$, and $\epsilon=0.7$. The spacings fall alternatingly onto one or the other of two smooth curves near-degeneracy as in Fig. 4.

yields a spacing distribution of the form

$$
\bar{P}_{2}(\Delta E)=\frac{\Theta\left(\Delta E_{\max }-\Delta E\right) \Theta\left(\Delta E-\Delta E_{\min }\right)}{\pi \sqrt{\left(\Delta E_{\max }-\Delta E\right)\left(\Delta E-\Delta E_{\min }\right)}} .
$$

It is equivalent to the density of states of a cosine-like dispersion branch with a gap in a $1 \mathrm{D}$ system. The actual

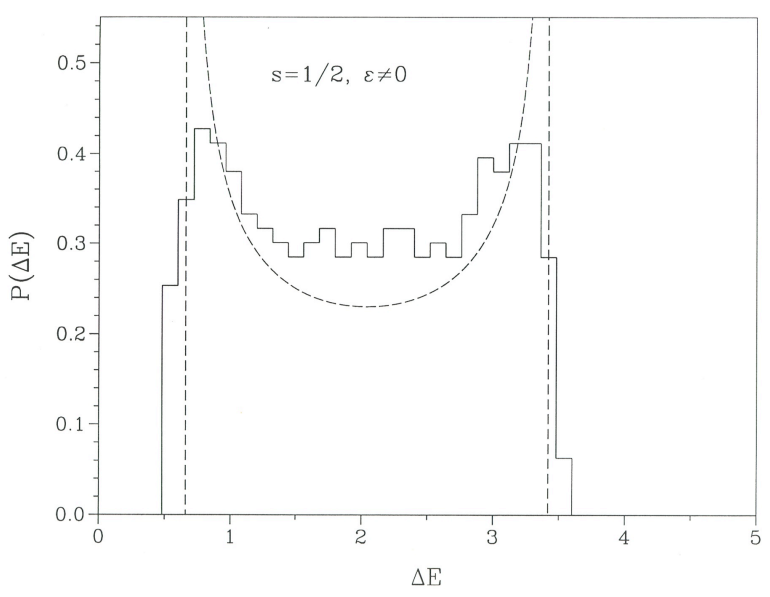

FIG. 6: Level-spacing distribution of the states shown in Fig. 4 , but over the extended energy range $100 \leq E_{\lambda} \leq 1130(515$ levels), of the (nonintegrable) $s=1 / 2$ spin-boson model (1) with $\hbar=1 / s=2, \omega_{B}=\omega_{S}=1, \Lambda=1$, and $\epsilon=0.7$ (histogram). The dashed line represents the distribution (5), which is predicted by a simple phenomenological model as described in the text. 
level-spacing distribution for this nonintegrable $s=1 / 2$ case is plotted as a histogram in Fig. 6. The characteristic properties which distinguish it from that of the integrable case are the complete absence of very small spacings and the marked enhancements near the band edges. Both properties are reasonably well represented by the model distribution (5), which is shown dashed in Fig. 6 with fitted parameter values. A more realistic model should take into account the slow amplitude modulations in the curves of Fig. 5. Level-spacing sequences and distributions for the $s=1 / 2$ spin-boson model were previously investigated by Kuś [8], but only for the case $\epsilon=1$ and with no conclusive evidence for quantum nonintegrability effects.

\section{SPIN $s=1$}

No matter how compelling the nonintegrability effects are that we have identified in the $s=1 / 2$ spin-boson model, it would be incorrect to see in them already manifestations of quantum chaos, but the resonance between nearly degenerate energy eigenstates represents an important building block thereof. The same basic ingredient comes into play also in the $s=1$ spin-boson model. Here it produces effects of increased complexity including disorderly level repulsion, which is a characteristic signature of quantum chaos.

\section{A. $[H, I]=0$}

The spectrum $\left(\left\langle a^{\dagger} S_{-}\right\rangle_{\lambda}, E_{\lambda}\right)$ of the integrable $s=1$ spin-boson model consists of three strands of levels as shown in Fig. 7. The two natural quantum numbers $\mu=-1,0,+1$ and $\nu=0,1,2, \ldots$ are suggested by the smoothly varying separation between strands and by the nearly uniform intra-strand level spacings, respectively, and also by the fact that states belonging to different strands do not resonate. Three occurrences of nearly degenerate levels can be observed in the inset to Fig. 7 .

Upon projection of the three strands of states onto the energy axis, the level spacings fall cyclically onto one of three nearly periodic curves as shown in Fig. 8 . The short-dashed curve describes spacings between states from nearest-neighbor $(\mathrm{nn})$ strands at all energies. The other two curves change slope midway between a maximum and a minimum. Any portion above the bend describes spacings between states from nn-strands, and any portion below the bend spacings between states from next-nearest-neighbor (nnn) strands.

The spacing sequences of Fig. 8 yield the spacing distribution shown as a histogram in Fig. 9. It is significantly different from the $s=1 / 2$ case (Fig. 3). Within the given statistical uncertainties, the histogram is con-

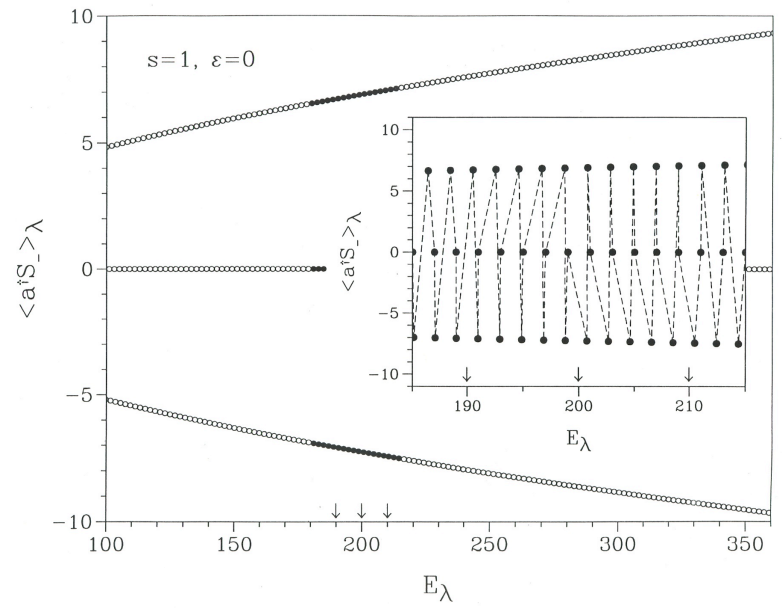

FIG. 7: Invariant $\left\langle a^{\dagger} S_{-}\right\rangle_{\lambda}$ versus energy eigenvalue $E_{\lambda}$ over a certain energy range for the eigenstates of parity +1 of the (integrable) $s=1$ spin-boson model (1), with $\hbar=1 / s=$ $1, \omega_{B}=\omega_{S}=1, \Lambda=1$, and $\epsilon=0$. A subset of these states (full circles) is shown again in the inset on an expanded energy scale and with consecutive levels connected by dashed lines. The arrows mark three successive locations of neardegeneracies between levels of different strands.

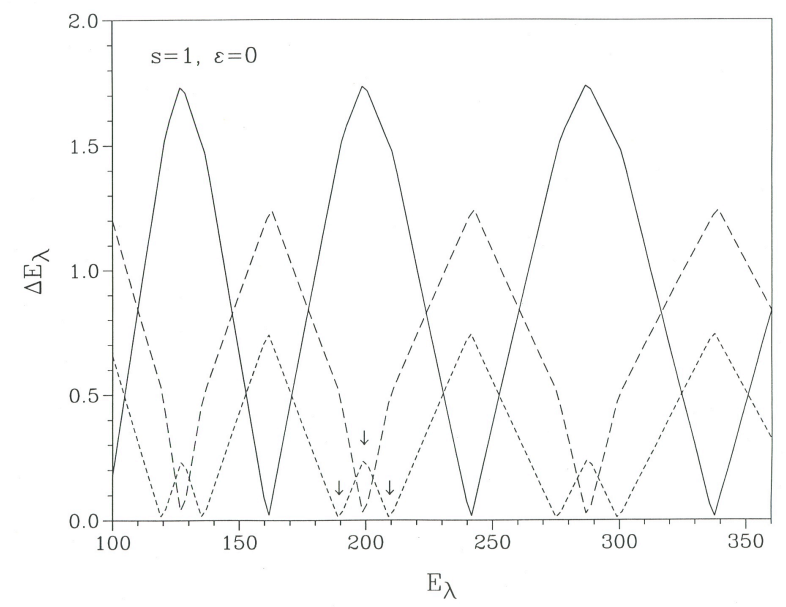

FIG. 8: Sequence of successive spacings $\Delta E_{\lambda}=E_{\lambda}-E_{\lambda-1}$ between the energy levels shown in Fig. 7 for the (integrable) $s=1$ spin-boson model (1), with $\hbar=1 / s=1, \omega_{B}=\omega_{S}=$ $1, \Lambda=1$, and $\epsilon=0$. The spacings fall cyclically onto one of three piecewise smooth curves (shown by long-dashed, shortdashed and solid lines). The arrows mark the same interstrand near-degeneracies as in Fig. 7.

sistent with the triangular distribution

$P_{3}(\Delta E)=\frac{2}{\Delta E_{S}}\left(1-\frac{\Delta E}{\Delta E_{S}}\right) \Theta(\Delta E) \Theta\left(\Delta E_{S}-\Delta E\right)$,

which is shown dashed for a fitted value of the band width $\Delta E_{S}$. 


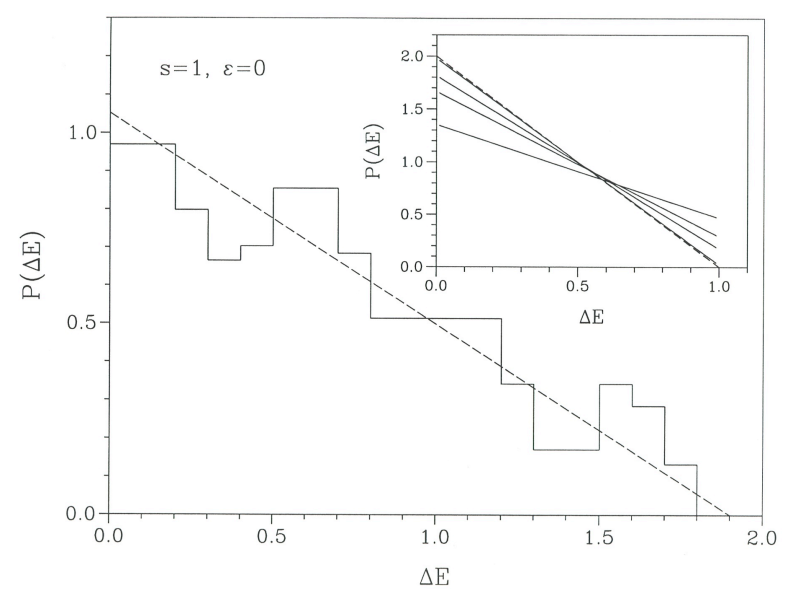

FIG. 9: Level-spacing distribution of the states shown in Fig. 7 , but over the extended energy range $32.5 \leq E_{\lambda} \leq 377.0$ (515 levels), of the (integrable) $s=1$ spin-boson model (1) with $\hbar=1 / s=1, \omega_{B}=\omega_{S}=1, \Lambda=1$, and $\epsilon=0$ (histogram). The dashed line represents the triangular distribution (6), which is predicted by a simple phenomenological model described in the text as a limiting case. The inset illustrates how that distribution varies with the parameter $\eta$ of that phenomenological model. The four solid lines in the order of increasing intercept pertain to $\eta=0.5,0.2,0.1,0.01$ and the dashed line represents the limiting function $\tilde{p}_{3}(x)=2(1-x)$.

This function is obtained as a limiting case from the lattice model for level configurations which we have already used in Sec. III.A. Consider the superposition of three 1D lattices with incommensurable lattice spacings $b_{1} \equiv b<b_{2}<b_{3}$. The resulting distribution of spacings $x$ between successive sites is then of the form

$$
p_{3}(x)=\left(1-u_{3}\right) \tilde{p}_{3}(x) \Theta(x) \Theta(b-x)+u_{3} \delta(x-b),
$$

where the continuous part $\tilde{p}_{3}(x)$ and the fraction of intralattice spacings $x=b$ depend on the values of $b_{2}$ and $b_{3}$. We have performed a numerical analysis of this lattice model based on $5 \times 10^{6}$ consecutive spacings for $b_{1}=$ $1, b_{2}=1+\eta \sqrt{2}, b_{3}=1+\eta \sqrt{3}$ and a range of $\eta$-values. The conclusions from that analysis are as follows:

(i) The weight of the $\delta$-function in (7) grows more slowly than in the $s=1 / 2$ case: $u_{3} \sim \eta^{2}$ for $\eta \ll 1$.

(ii) The continuous part of (7) is trapez-shaped as shown in the inset to Fig. 9 and approaches the triangular function $\tilde{p}_{3}(x)=(2 / b)(1-x / b)$ for $\eta \rightarrow 0$.

(iii) This limiting distribution is universal, i.e. it obtains for arbitrary incommensurable lattice spacings $b_{1}<$ $b_{2}<b_{3}$ with very small differences.

\section{B. $[H, I] \neq 0$}

The impact of nonintegrability on the three strands of eigenstates depicted in Fig. 7 for the $s=1$ case shares

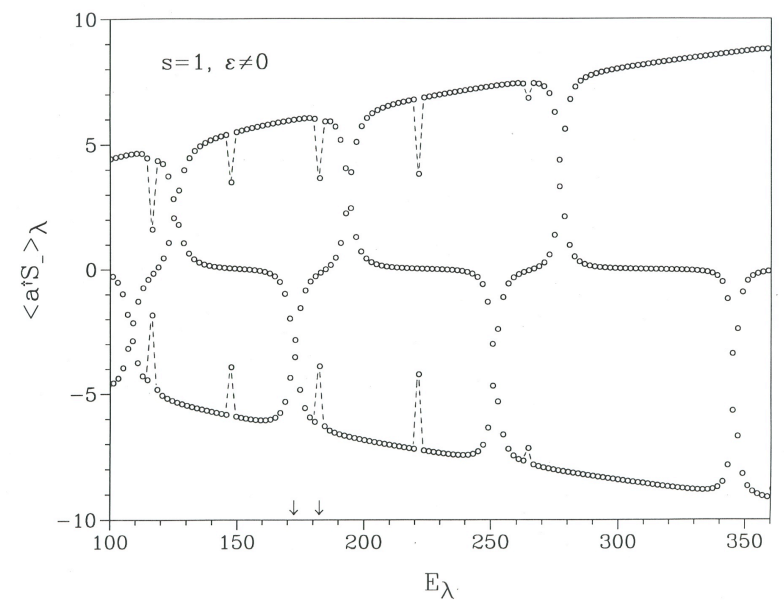

FIG. 10: Invariant $\left\langle a^{\dagger} S_{-}\right\rangle_{\lambda}$ versus energy eigenvalue $E_{\lambda}$ over a certain energy range for the eigenstates of parity +1 of the (nonintegrable) $s=1$ spin-boson model (1), with $\hbar=1 / s=1, \omega_{B}=\omega_{S}=1, \Lambda=1$, and $\epsilon=0.4$. The arrows mark the locations of one near-degeneracy between states from nearest-neighbor strands (left) and one between states from next-nearest-neighbor strands (right).

many attributes with the $s=1 / 2$ scenario, but there are additional features. States from nn-strands are undergoing resonances at fairly regular intervals. This causes the three strands to be twisted into a single braid, as shown in Fig. 10. In the $s=1 / 2$ case, nn-resonances were the only nonintegrability effect. Here, in the $s=1$ case, they remain dominant, but the additional possibility of resonances between states from strands that are not adjacent in the $\left(\left\langle a^{\dagger} S_{-}\right\rangle_{\lambda}, E_{\lambda}\right)$-plane (nnn-resonances) moves the system one step closer to quantum chaos. These nnnresonances, several of which can be observed in Fig. 10, introduce a certain amount of disorder into the picture that was totally absent in the $s=1 / 2$ case. They are generally weaker than nn-resonances in the sense that two states must be closer in energy to produce a comparable effect on the matrix element $\left\langle a^{\dagger} S_{-}\right\rangle_{\lambda}$. The amount of level repulsion is correspondingly smaller between states from nnn-strands.

The different degrees of level repulsion are best demonstrated in Fig. 11, which depicts the sequence of level spacings $\Delta E_{\lambda}$ for the states of Fig. 10. As in Fig. 8 (integrable case), the spacings fall onto three nearly periodic curves with similar properties. The short-dashed curve describes spacings between states from nn-strands only, while the solid and dashed lines describe nnn-spacings on any stretch below the bend and nn-spacings above the bend. All nn-resonances observable in Fig. 10 coincide with a minimum of the short-dashed line in Fig. 11. One occurrence is marked by an arrow (left) in both plots. These minima are nonzero by a significant amount similar to the ones observes in Fig. 5 for the $s=1 / 2$ case.

The spacings between nnn-strands, on the other hand, 


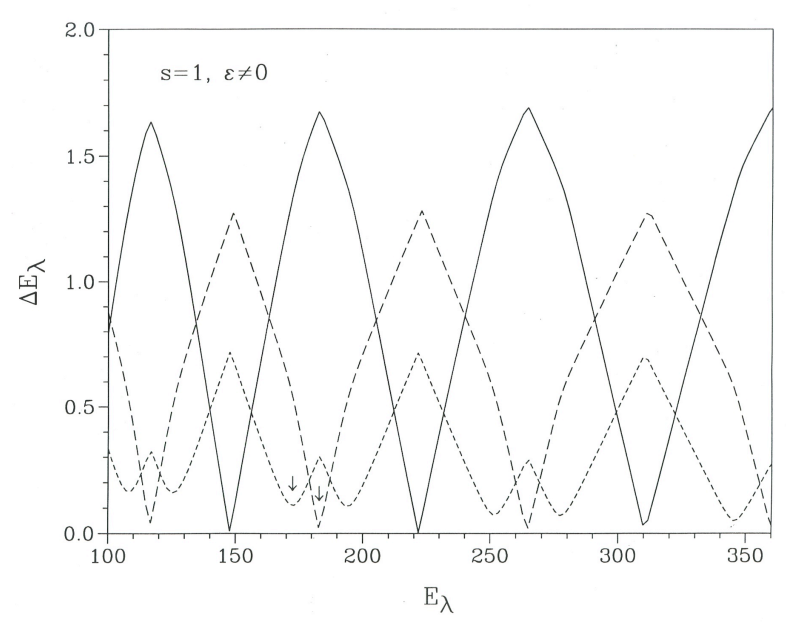

FIG. 11: Sequence of successive spacings $\Delta E_{\lambda}=E_{\lambda}-E_{\lambda-1}$ between the energy levels shown in Fig. 10 for the (nonintegrable) $s=1$ spin-boson model (1), with $\hbar=1 / s=1, \omega_{B}=$ $\omega_{S}=1, \Lambda=1$, and $\epsilon=0.4$. The spacings (circles) fall cyclically onto one of three piecewise smooth curves (shown by long-dashed, short-dashed and solid lines). The arrows mark the same inter-strand near-degeneracies as in Fig. 10.

experience a degree of level repulsion that is imperceptly small on the scale of Fig. 11. One nnn-resonance is marked by an arrow (right). The spacing sequence of Fig. 11 yields the spacing distribution shown in Fig. 12. The probability of very small spacings is considerably suppressed with respect that found in the integrable case (Fig. 9), but that suppression is far less complete than that observed in the $s=1 / 2$ case (Fig. 6).

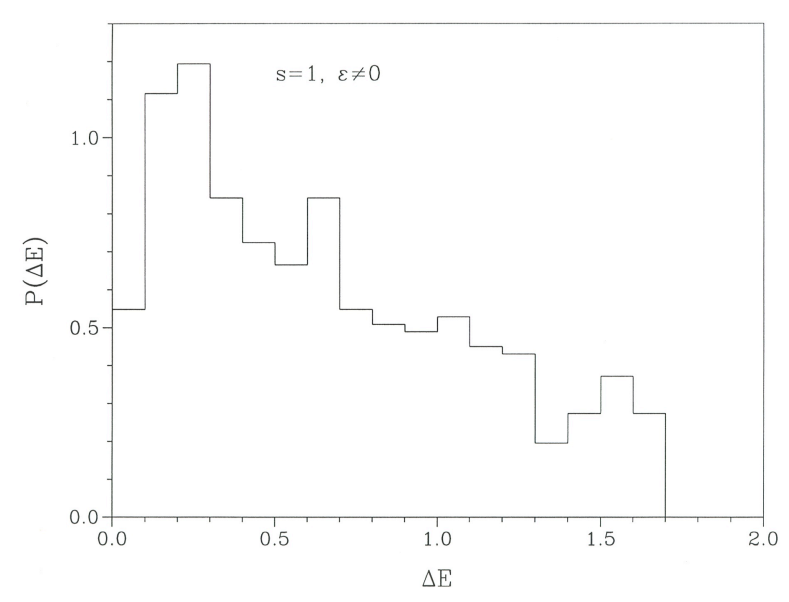

FIG. 12: Level-spacing distribution of the states shown in Fig. 10, but over the extended energy range $32.5 \leq E_{\lambda} \leq 377.0$ (515 levels), of the (nonintegrable) $s=1$ spin-boson model (1) with $\hbar=1 / s=1, \omega_{B}=\omega_{S}=1, \Lambda=1$, and $\epsilon=0.4$.

\section{V. $\quad$ SPIN $s=3 / 2, \ldots$}

The phenomena described in the Secs. III and IV for the $s=1 / 2$ and $s=1$ spin-boson model have all been based on two elements: (i) the general multi-strand structure of the spectrum, (ii) the presence of inter-strand resonances for $\epsilon \neq 0$. In systems with as few as two or three strands of states, these elements give rise to special levelspacing distributions, which we have been able to analyse and interpret quantitatively, at least to a certain extent.

Our numerical evidence suggests that the same picture obtains for arbitrary spin quantum numbers. Fig. 13 shows the four well-separated strands of states in the $\left(\left\langle a^{\dagger} S_{-}\right\rangle_{\lambda}, E_{\lambda}\right)$-plane for the integrable $s=3 / 2$ spinboson model (dotted lines). As the spin-boson coupling is made nonintegrable, the nn-resonances cause the four strands to form the familiar braid. The resulting configuration is further perturbed by nnn-resonances. Furtherneighbor resonances are too weak to be discernible in this plot.

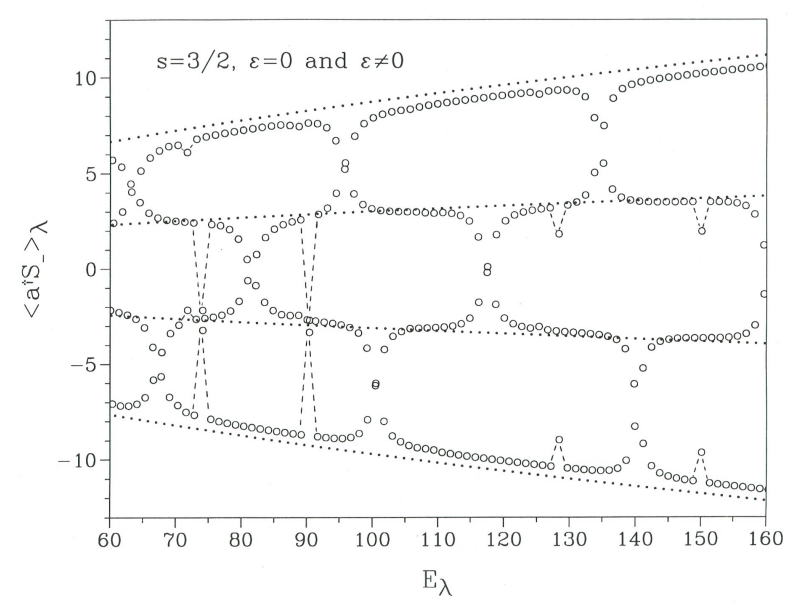

FIG. 13: Invariant $\left\langle a^{\dagger} S_{-}\right\rangle_{\lambda}$ versus energy eigenvalue $E_{\lambda}$ over a certain energy range for the eigenstates of parity +1 of the (nonintegrable) $s=3 / 2$ spin-boson model (1), with $\hbar=1 / s=$ $2 / 3, \omega_{B}=\omega_{S}=1, \Lambda=1.5$ and with $\epsilon=0$ (integrable case, dots) and $\epsilon=0.4$ (nonintegrable case, open circles).

The level-spacing distribution inferred from the four strands of states of the integrable $s=3 / 2$ spin-boson model is shown as a histogram in Fig. 14. In spite of the statistical fluctuations, we can see that this result is one step closer to the Poisson distribution than the $s=1$ result of Fig. 9 and two steps closer than the $s=1 / 2$ result of Fig. 3 . The Poisson distribution is the default expectation for a generic integrable model with two degrees of freedom in the semiclassical regime.

What are the predictions of our simple lattice model for the general spin-s case of the integrable spin-boson model? Consider the superposition of $n=2 s+11 \mathrm{D}$ lattices with incommensurable lattice spacings $b_{1} \equiv b<$ $b_{2}<\ldots<b_{n}$. The general form of the spacing dis- 


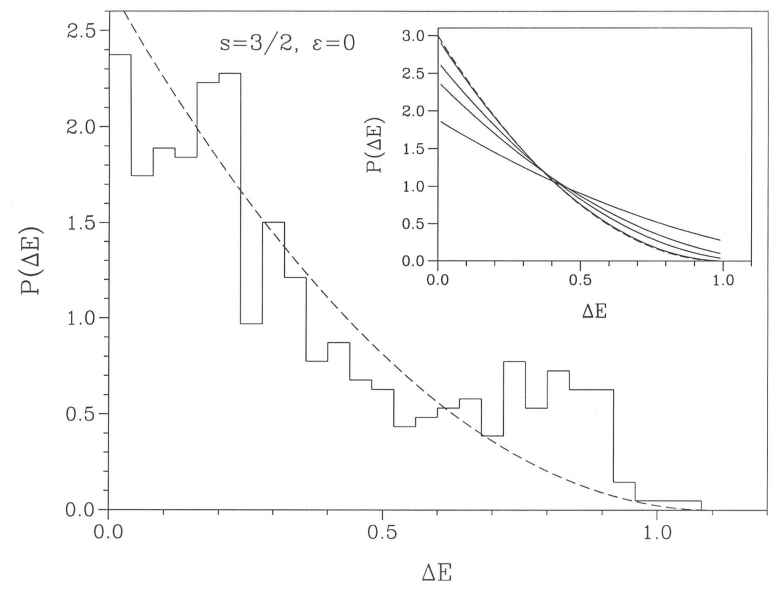

FIG. 14: Level-spacing distribution of the states shown in Fig. 13, but over the extended energy range $16 \leq E_{\lambda} \leq 189$ (515 levels), of the (integrable) $s=3 / 2$ spin-boson model (1) with $\hbar=1 / s=2 / 3, \omega_{B}=\omega_{S}=1, \Lambda=1.5$ and with $\epsilon=0$ (histogram). The inset illustrates how the continuous part $\tilde{p}_{4}(x)$ of the distribution (8) of the phenomenological lattice model described in the text varies with the parameter $\eta$. The four solid lines in the order of increasing intercept pertain to $\eta=0.5,0.2,0.1,0.01$ and the dashed line represents the limiting function $\tilde{p}_{4}(x)=(3 / b)(1-x / b)^{2}$ with $b=1$. The same function with $b$ adjusted to fit the histogram is shown dashed in the main plot.

tribution is found to be the same as we have already established for $s \leq 1$ :

$$
p_{n}(x)=\left(1-u_{n}\right) \tilde{p}_{n}(x) \Theta(x) \Theta(b-x)+u_{n} \delta(x-b) .
$$

The continuous function $\tilde{p}_{n}(x)$ is non-universal (i.e. dependent on $\left.b_{1}, \ldots, b_{n}\right)$ if $n \geq 3$. However, for very small differences in lattice spacings that dependence disappears.

The results of our numerical analysis of the $n=4$ case based on $5 \times 10^{6}$ consecutive spacings for $b_{1}=1, b_{2}=$ $1+\eta \sqrt{2}, b_{3}=1+\eta \sqrt{3}, b_{4}=1+\eta \sqrt{5}$, are displayed in the inset to Fig. 14 for a range of $\eta$-values. For $\eta \rightarrow 0$, the solid curves converge towards the dashed curve, which represents the limiting function $\tilde{p}_{4}(x)=(3 / b)(1-x / b)^{2}$ with $b=1$. The same function with $b$ adjusted fits the spin-boson data reasonably well (see dashed line in the main plot of Fig. 14).

The continuous part $\tilde{p}_{n}(x)$ of the spacing distribution (8), which is universal for very small differences in lattice spacings, is found to be the following general expression [14]:

$$
\tilde{p}_{n}(x)=\frac{n-1}{b}\left(1-\frac{x}{b}\right)^{n-2} .
$$

As $\eta \rightarrow 0$, the mean level spacing becomes $\bar{x}=b / n$. After the variable change $x^{\prime}=x / \bar{x}$ in $\tilde{p}_{n}(x) d x=\tilde{p}_{n}^{\prime}\left(x^{\prime}\right) d x^{\prime}$ we obtain, in the limit $n \rightarrow \infty$, the Poisson distribution $\tilde{p}_{\infty}^{\prime}\left(x^{\prime}\right)=e^{-x^{\prime}}$, as is expected. The limiting case of superimposed 1D lattices was previously discussed in Ref. [15].

Our method of analysis has thus yielded a fairly comprehensive picture of the $s$-dependent level statistics for the integrable spin-boson model. In addition to that, we have demonstrated that nonintegrability effects can be identified and described in quantitative terms even for the smallest spin quantum numbers.

What has yet to be accomplished is the construction of a modified lattice model for the nonintegrable cases of the spin-boson model - a model that is again based on the multi-strand spectrum, but that takes also into account inter-strand resonances. Our only result in that category thus far is expression (5) for the $s=1 / 2$ case.

\section{Acknowledgments}

The research done at URI was supported by the U.S. National Science Foundation, Grant DMR-93-12252. Y.C. has been supported by the Swiss Foundation for Scientific Research. M.C. is grateful to R. Livi and A. Politi for their kind hospitality during his stays at the INO. The computations were initiated at the Institut de Physique de l'Université de Neuchâtel (Switzerland) and jointly carried out at the EPFL, the INO and the National Center for Supercomputing Applications, University of Illinois at Urbana-Champaign.

\section{References}

1. Graham, R., Höhnerbach, M.: Z. Phys. B 57, 233 (1984)

2. Cibils, M., Cuche, Y., Wreszinski, W.F., Amiet, J.P., Beck, H.: J.Phys. A 23, 545 (1990)

3. Cibils, M., Cuche, Y., Leboeuf, P., Wreszinski, W.F.: Phys. Rev. A 46, 4650 (1992)

4. Müller, L., Stolze, J., Leschke, H., Nagel, P.: Phys. Rev. A 44, 1022 (1991)

5. Aguiar, M., Furuya, K., Levenkopf, C., Nemes, M.: Europhys. Lett. 15, 125 (1991) and Ann. Phys. 216, 313 (1992)

6. Cibils, M., Cuche, Y., Marvulle, V., Wreszinski, W.F., Amiet, J.P., Beck, H.: J. Phys. A 24, 1661 (1991)

7. Lewenkopf, C., Nemes, M., Marvulle, V., Pato, M., Wreszinski, W.F.: Phys. Lett. A 155, 113 (1991)

8. Kuś, M.: Phys. Rev. Lett. 54, 1343 (1985)

9. Srivastava, N., Müller, G.: Phys. Lett. A 146, 282 (1990) and Z. Phys. B 81, 137 (1990) 
10. Srivastava N., Kaufman, C., Müller, G.: J. Appl. Phys. 67, 5627 (1990)

11. Reichl, L.: The transition to chaos in conservative classical systems: quantum manifestations. New York: Springer 1992, pp. 234-246.

12. Aguiar, M., Furuya, K., Lewenkopf, C., Nemes, M.: Ann. Phys. 216, 291 (1992)

13. Cuche, Y.: Etude du chaos quantique dans le modèle spin-boson. (Thèse de doctorat, Université de Neuchâtel 1992)

14. Cuche, Y.: private communication (unpublished)

15. Haake, F.: Quantum signatures of chaos. Berlin: Springer 1991, Sec. 5.5 